\section{A CASE OF ABSCESS OF THE BRAIN.*}

BY JOHN K. SPENDER, M.D.LOND.,

Surgeon to the Mineral Water Hospital, and to the Eastern Dispensary, Bath.

THE following case possesses some interest and value, on account of the complete history which was obtained. Some points in that history, too, may help to place the diagnosis of cerebral abscess on a surer foundation.

G. T., a well-built muscular man, aged 60 , by occupation a carter, and in apparently sound and even vigorous health, fell off a load of thorns in the middle of November 1866, and struck the back of his head. He was severely shaken, but was not made insensible. He did not vomit; and there was no outward injury inflicted on the body, beyond bruises. He stayed at home quietly for four days, and then went to work again ; but, for many days after this, he always came home early, and, in a silent moody way, sat shivering all the evening over the fire. He often went to bed as early as seven o'clock, and said that he had a noise in his head like "singing". All through January, he stayed at home entirely, and was always silent and strange. The people around him had to speak two or three times before they could get an answer. This went on through February and nearly the whole of March. On March 28th, he felt so much better, that he went to a wood with two or three other men, in order to fell trees; and, while doing this, he had a "fit"-strictly epileptiform, for he was insensible and con. vulsed, and was brought home by his companions. Next morning, he was so far revived, that he resumed the work of the previous day, and did so until Monday, April rst, when he came home about noon, and did not go out again all the week. It was on Wednesday, April 3 rd, that he was seen for the first time by the house-surgeon of the Eastern Dispensary, Mr. Exell. On Sunday evening, April 7 th, he was remarkably lively and cheerful. The next day, he felled trees again in the old wood, but was again brought home in an epileptic convulsion, and never afterwards went out of his bed-chamber.

From this time till the middle of June, he received parochial relief and medical attendance. He was always in bed; and, at ten o'clock every morning, he was seized with a violent tremor, followed by a pungent heat and a profuse sweating; and, strange to relate, this hectic cycle was almost always most intense on Tuesdays. He now began to complain of an intense screwing pain at the vertex of the right half of the head, which continued for nearly three months with cruel rigour. A letter for the Fastern Dispensary was obtained for him in June, and I visited him rather frequently.

No considerable variation in the symptoms occurred in July; but, in the first week of August, he suffered another, a third and last, epileptic fit. After this, he had no more pain; but he now lost his speech, and could utter nothing beyond groaning inarticulate noises. At about the same time, the power of swallowing also was lost. The pupils were always normal, and responded well to light. The protruded tongue invariably turned to the left side. Paralysis of the left arm and leg gradually came on-the arm and leg which were clonically convulsed during the epileptic attacks; and the ever-spreading palsy soon merged into a coma which deepened into death, on Saturday morning, September 14 th.

Post Mortem Examination, Sunday evening, Sept. 15th. -I obtained this examination on condition that the head should be the only cavity opened. There was no scar visible on any part of the scalp. The calvarium was removed without difficulty, and there were no adhesions between it and the dura mater. The membranes had a congested look over all the upper surface of the brain; and the right hemisphere seemed swollen, much in the same way as the brain of a child who has died of acute tubercular meningitis. Slicing the brain carefully down, I came, at about half an inch from the surface, to the roof of a large and irregularly shaped cavity, lined with a soft membrane, and out of which a thin puriform fluid so suddenly poured that I was unable to collect it for measurement. The body of the cavity might have contained a plum of moderate size. A sinus ran half an inch directly forwards; and another and much longer one took a direction downwards and inwards into the lateral ventricle, into which pus of a thicker consistence had filtered. So far as I could make out, the right lateral ventricle formed the lower and inner boundary of the purulent cavity. The cerebral tissue around the abscess was deeply reddened. The entire brain was next removed; and the bones at the base of the brain were carefully inspected; so also were the principal blood-vessels; but nothing wrong could be discovered. During life, the organs of sense had done their duty well; and physical examination of the other cavities of the body gave no indication of disease, though it ought to be added that, during the epileptic convulsions, the heart palpitated in a most violent manner.

- Read before the Bath and Bristol Branch.
The treatment of this case is a confession of the impotency of medicine. Everything reasonable was tried: iodide of potassium in large doses; a short and mild course of mercury; a seton at the back of the neck. Only opium produced transient and imperfect remission of sufof swallowing it.

The history of this case embodies the striking fact, that there. was no apparently adequate cause for the mischief in the brain. He was much. shaken by falling on the head; but he was not wounded. No outward phlegmon had been seen or known during the last few years of life; and, if the man's statement is to be believed, there had been no syphilis for at least thirty years. The health had been unqualifiedly good for a long time, though he occasionally drank rather hard. There was no albumen in the urine when I attended him.

Dr. Gull's monograph on Abscess of the Brain, in the third volume of the third series of Guy's Hospital Reports, is of high classical value. Relying on his almost absolute assertion that, "with the exception of suppuration produced by scrofulous deposit, idiopathic abscess of the brain does not occur," I hesitated in offering a perfect diagnosis, and suspected for some time that my patient was an example of chronic cerebral soitening. The local pain and the unilateral convulsion and paralysis helped me at length to realise the actual state of things. It is noticeable how the earlier symptoms, indicating pressure on the surface of the convolutions, receded to make way for the abolition of the mental and motorial faculties, which must have been caused by pressure on the dcep-seated ganglia. In these facts, we have a key to the new direction of the pus-containing sinuses. Dr. Gull contends that a scrutiny of every organ of the body is necessary in order to fix the exact etiology of any case of abscess of the brain; but so exhaustive and laborious a necropsy is not within every one's power; and Lebert distinctly admits the possibility of "idiopathic cerebral suppuration".

\section{CASES OF INTESTINAL OBSTRUCTION; WITH REMARKS.*}

\author{
BY WILLIAM CADGE, F.R.C.S., \\ Surgeon to the Norfolk and Norwich Hospital.
}

Every case of Intestinal Obstruction, threatening to be, or being, invincible from any cause, is of such interest to the surgeon, and is fraught with such danger to the patient, that I shall make no apology for occupying the time of the meeting while I narrate and comment on a
very few of the many cases which have come under my notice; and I shall only mention those which present some feature of surgical or pathological interest.

Before alluding to cases of complete, I would briefly note the occurrence of cases of partial, obstruction. In many of the former, there is a period, of variable duration, during which the patient has symptoms like the following : frequent bilious attacks, so-called-that is, vomiting, abdominal pain and distension, and more or less constipation. Now these very common symptoms would not usually attract much attention; nor need they, if they do not frequently recur; but if they are of frequent occurrence, it is of essential importance to examine carefully the abdomen for some mechanical explanation. A short time ago, I saw a gentleman, who had had these frequent bilious attacks; and, when I carefully examined him in the recumbent position, with the knees drawn up, I could detect a hard tumour of the size of one's fist, on the right side of the umbilicus, deeply placed amongst the intestines. I could not ascertain either its nature or its exact connexions; but it was clearly the cause of occasional partial stoppage to the passage of the intestinal
contents. It never led to complete obstruction; but he died worn out with these repeated "bilious attacks."

In another instance, which I watched for two or three years, and which I have put on record, the early attacks were similar to those I have described, and were thought to be due to gout or over-indulgence, for the patient was a country gentleman who "fared sumptuously every day"; but, with the greatest care over diet, the attacks still recurred. He would frequently vomit large quantities of the contents of the small intestines; there was great distension of the abdomen and increased peristaltic movements; sometimes the vomiting was relieved by an attack but he never suffered complete obstruction.

On post mortem exámination, I found a fibrous tumour in the mesentery, which dragged the small intestine so much out of the course as to form a sort of elbow, and as to partially obstruct the passage of its con-

- Read at the meeting of the East Anglian and Cambridge and Funtingdon Branches. 